\title{
COLLUSION OR SNIPING IN SIMULTANEOUS ASCENDING AUCTIONS - A PRISONER'S DILEMMA
}

\author{
SASCHA FÜLLBRUNN \\ University of Luxembourg \\ Faculty of Law, Economics and Finance \\ Luxembourg School of Finance \\ 4, Rue Albert Borschette, L-1246 Luxembourg \\ sascha.fullbrunn@uni.lu \\ http://<sascha.fuellbrunn.de>
}

\begin{abstract}
In simultaneous ascending price auctions with heterogeneous goods Brusco and Lopomo [2002] derive collusive equilibria, where bidders divide objects among themselves, while keeping the prices low. Considering a simultaneous ascending price auction with a fixed deadline, i.e. the hard close auction format, a prisoner's dilemma situation results and collusive equilibria do not longer exist, even for only two bidders. Hence, we introduce a further reason for sniping behavior in Hard Close auctions, i.e. to appear to collude early in the auction and to defect at the very last moment.
\end{abstract}

Keywords: Collusion; multi unit auctions; prisoners's dilemma.

Subject Classification: D44

\section{Introduction}

Brusco and Lopomo [2002] (BL) consider collusive behavior in simultaneous ascending price auctions, i.e. in English auctions which are conducted in parallel (see also Engelbrecht-Wiggans and Kahn [2005]). Assuming private values, they derive a symmetric perfect Bayesian equilibrium in which the bidders split the markets by signaling their favorite market, even if communication is not allowed. ${ }^{\text {a }}$ Therefore, a "collusive agreement" holds due to the punishment mechanism, because defection leads to a "bidding war". b The hard close auction format, i.e. an English auction which is limited in time as on eBay.com, disables the punishment mechanism. We show that in hard close auctions collusive behavior is dominated by a sniping

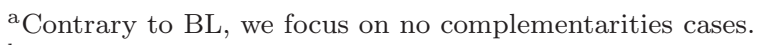

${ }^{\mathrm{b}}$ Kwasnica and Sherstyuk [2007] test this theoretical consideration experimentally and conclude that "outcomes of these auctions, when classified as collusive, often match the above-mentioned signaling model quite well".
} 
strategy, i.e. appearing to collude early in the auction and defecting at the very last moment. ${ }^{\mathrm{c}}$

In the following section, we introduce bidding behavior in the auction formats being considered for a single object in an online auction framework. Section 3 sketches the findings of BL and provides an analoguous approach in the hard close auction format. Finally, we conclude.

\section{Separated Auctions}

We compare bidding behavior using the framework of a soft close auction, the "online" version of the English auction, and the hard close auction. In both cases, we implement a bidding agent in the model according to the eBay pricing rule used in Ockenfels and Roth [2006]. Bidders submit a "maximum amount" that they are willing to pay for the object. The bidding agent overbids all other bids "up to the maximum bid", i.e. the agent "increases the bid by increments only as much as necessary to maintain a high bid position". ${ }^{\mathrm{d}}$ Keeping the increment as small as possible (zero), we model the auctions as dynamic second-price formats with discrete bidding stages (Füllbrunn and Sadrieh [forthcoming]).

Hence, at any time $t$, the current price equals the second highest bid submitted during the previous stage. The current holder at time $t$ is the bidder who has submitted the highest bid. At each stage, bidders are informed on the current price and on their status as current holders. After the auction, the current holder receives the objects and pays second highest bid. In case of a tie, the holder is determined randomly with equal probability amongst the high bidders and the price equals the highest bid. We consider a private value environment and the payoff of a buyer equals the difference between her valuation and the price. Other bidders receive no payoff. Since the auction is symmetric, private values are identically and independently drawn from a common distribution which is common knowledge. Bidders cannot observe the other bidders' values, face no liquidity constraints and are risk neutral.

The hard close auction consists of $T$ stages. The soft close auction consists of at least $T$ stages. If no bid is submitted in $T$, the auction ends. As long as bids are submitted in stages $T, T+1, \ldots$ a further stage occurs. In this case the auction ends after a stage without a bid. This framework has the same features as the English auction, i.e. the bidding agents play the equilibrium strategy of the English auction.

Due to the bidding agent framework, the terminal stage in both formats equals a sealed bid second-price auction, where the weakly dominant strategy is to submit a bid that equals the valuation (Vickrey [1961]). Bids below valuations in previous

'Sniping is a widely accepted stylized fact and has several reasons: sniping prevents incremental (Wintr [2008]) and shill bidding (Engelberg and Williams [2009]), it prevents the revealing of information during early stages (Rothkopf et al. [1990]) or is due to uncertainty over one's own private valuation (Rasmusen [2006]). See Ockenfels et al. [2006] for an overview.

d See http://pages.ebay.com/help/buy/bidding-overview.html\#incremental how it works on eBay. 
stages are arbitrary. Bids above valuations bear a risk of paying more for the object than the object is worth. Hence, in equilibrium the bidder with the highest valuation receives the object and pays a price that equals the second highest valuation. ${ }^{\mathrm{e}}$ "Sniping", i.e. bids according to the weakly dominant strategy for the first time in $T$, remains a reasonable strategy only in the hard close auction. However, with private values the revenue equivalence theorem holds and the ex ante revenues are the same in both auction formats (Myerson [1981]).

\section{Simultaneous Auctions}

In our model, two risk neutral bidders $(i=x, y)$ compete for two heterogeneous goods $(j=1,2)$. The bidders' valuation for each good, $v_{i j}$ is identically and independently drawn from the same probability distribution with limits 0 and 1 , density $f(v)$ and c.d.f. $F(v)$. As in BL (p. 414), we assume $E(v):=\int_{0}^{1} \leq \frac{1}{2}$. Without this assumption, both objects can be obtained at a low expected price, even without colluding. The payoff function for bidder $x$ depending on final bids $\beta_{x 1}, \beta_{x 2}$ and $\beta_{y 1}, \beta_{y 2}$ is (respectively for bidder $y$ )

$$
\pi_{x}=\left\{\begin{array}{clll}
v_{x 1}-p_{1}+v_{x 2}-p_{2}, & \text { if } \beta_{x 1}>\beta_{y 1} & \text { and } & \beta_{x 2}>\beta_{y 2} ; \\
v_{x 1}-p_{1}, & \text { if } \beta_{x 1}>\beta_{y 1} & \text { and } & \beta_{x 2}<\beta_{y 2} \\
v_{x 2}-p_{2}, & \text { if } \beta_{x 1}<\beta_{y 1} & \text { and } & \beta_{x 2}>\beta_{y 2} \\
0, & \text { if } \beta_{x 1}<\beta_{y 1} & \text { and } & \beta_{x 2}<\beta_{y 2}
\end{array}\right.
$$

where $p_{j}$ is the price in the respective market. The bidders can submit bids in two simultaneous but separated dynamic second-price auctions. In order to keep things simple we assume $T=2$. In hard close auctions, both markets simultaneously start with stage 1 and end with stage 2 . Thus, the bidders can submit only two bids in each market. In soft close auctions, both markets simultaneously start with stage 1 and simultaneously end either after stage 2, if no bid is submitted in stage 2 in either market, or after stage $2+s$, if bidders submit bids in any stage $2,3, \ldots 2+s-1$ but neither bids in stage $2+s$.

The lowest bid is $b_{0}=0$ and the bidders have the possibility not to bid, i.e. they bid $\omega$. A bid in stage $t$ by bidder $i$ in market $j$ is $b_{i j}^{t}$.

\subsection{The simultaneous soft close auction}

In BL, proposition 0 claims that overbidding any price as long as positive payoffs are possible (SEA strategy) forms a perfect Bayesian equilibrium. ${ }^{\mathrm{f}}$ However, with some consistent beliefs, proposition 1 has established the existence of a symmetric perfect Bayesian equilibrium which dominates the perfect Bayesian equilibrium in

${ }^{\mathrm{e}}$ As is generally known, asymmetric equilibria in second price auctions exist. For example, only the bidder with the highest value submits a bid. However, we refer to equilibria with symmetric strategies.

${ }^{\mathrm{f}}$ We assume the bidders do not overbid their private valuation. 
SEA strategies in terms of bidders' surplus. ${ }^{\mathrm{g}}$ According to proposition 0, our bidding agent framework leads to a modification of the SEA strategy: we refer to the "SSC" strategy as finally submitting their own valuation in any market as maximum bids. The bidding agent then bids the next highest maximum bid in the respected market. However, the equilibrium results hold as in proposition 0 in BL.

Any simple collusion (CSC) strategy that is analoguous to Proposition 1 of BL is $\left(0 \leq b_{i j} \leq v_{i j}\right)$ :

- If $v_{i 1}>v_{i 2}$ submit $b_{i 1}^{1}=b_{i 1}$ and $b_{i 2}^{1}=\omega$;

- If $v_{i 1}<v_{i 2}$ submit $b_{i 1}^{1}=\omega$ and $b_{i 2}^{1}=b_{i 2}$;

- If, in stage 2 ,

$$
\begin{aligned}
& b_{x 1}^{1}=\omega \text { and } b_{y 1}^{1} \geq 0 \text { and } b_{x 2}^{1} \geq 0 \text { and } b_{y 2}^{1}=\omega \\
& \text { or } \\
& b_{x 1}^{1} \geq 0 \text { and } b_{y 1}^{1}=\omega \text { and } b_{x 2}^{1}=\omega \text { and } b_{y 2}^{1} \geq 0
\end{aligned}
$$

the bidders divide the markets among each other and take no further action in the following stages;

- if, in stage $2, b_{x j}^{1} \geq 0$ and $b_{y j}^{1} \geq 0$ or someone defects from the bidding instructions given above, then all types revert to the SSC strategy.

The bidders only submit one bid in the market with their higher valuation; and without competition in that market they take no further action later on. Note that due to the bidding agent any bid until the valuation can be used as a signal. In all other cases, the perfect Bayesian equilibrium in SSC strategies evolves. ${ }^{\mathrm{h}}$ Therefore, a special distribution of values is necessary to facilitate collusive results. Either $v_{x 1}>v_{x 2}$ and $v_{y 2}>v_{y 1}$ or $v_{x 1}<v_{x 2}$ and $v_{y 2}<v_{y 1}$ leads to a collusive result under the given circumstances. Due to symmetry, we assume $v_{x 1}>v_{x 2}$ and $v_{y 2}>v_{y 1}$ in the following.

In the proof of Proposition 1, BL conclude that given the conditions in 3 "the bidders will collude when the opportunity arises". This includes giving a correct signal in the first stage, i.e. bidders are willing to truthfully signal their type (the market with the higher valuation). Hence, for bidder $x$ the surplus using the CSC strategy equals $\pi_{x}(C S C)=v_{x 1}$. Defecting from the CSC strategy leads to the expected surplus (the results for bidder $y$ are analoguous):

$$
\begin{aligned}
E\left[\pi_{x}(S S C)\right]= & \int_{0}^{v_{x 1}}\left(v_{x 1}-v_{y 1}\right) 2 f\left(v_{y 1}\right)\left(1-F\left(v_{y 1}\right)\right) d v_{y 1} \\
& +\int_{0}^{v_{x 2}}\left(v_{x 2}-v_{y 2}\right) 2 f\left(v_{y 2}\right) F\left(v_{y 2}\right) d v_{y 2},
\end{aligned}
$$

\footnotetext{
gEngelbrecht-Wiggans and Kahn [2005] discuss sets of consistent beliefs of the bidders.

${ }^{\mathrm{h}} \mathrm{BL}$ also discuss collusion strategies with more information. For us it is sufficient to discuss the easiest form of collusion.
} 
which is lower than $\pi_{x}(C S C)$. The first part of the equation equals the expected payoff in market 1 given $v_{y 1}$ is the lower value of bidder $y$ and the second part equals the expected payoff in market 2 given $v_{y 2}$ is the higher value of bidder $y$.

The sellers' payoff is zero in the CSC case and positive in the SSC case. Furthermore, collusive behavior can decrease efficiency in the CSC case. Assume for example $v_{x 1}>v_{x 2}, v_{y 1}<v_{y 2}$, and $v_{x 2}>v_{y 2}$ : CSC strategies leave object 2 to bidder $y$ although bidder $x$ has a higher valuation for this object. SSC strategies always lead to efficient outcomes.

In the simultaneous soft close auction with two markets and two bidders collusive behavior is possible and forms a symmetric perfect Bayesian equilibrium in CSC strategies which dominates the perfect Bayesian equilibrium in SSC strategies in terms of bidders' surplus. However, the seller is worse off and efficiency is reduced.

\subsection{The simultaneous hard close auction}

As in part 3.1 we only consider a situation where collusion is possible $\left(v_{x 1}>v_{x 2}\right.$ and $\left.v_{y 2}>v_{y 1}\right)$. Further, we assume that bidders truthfully signal their preferred market in the first stage as discussed in the appendix of BL (p. 429).

We show that collusive behavior, especially a modification of the CSC strategy, cannot be an equilibrium strategy. ${ }^{\mathrm{i}}$ Therefore, we consider two signaling strategies, where bidders signal their preferred market in the first stage. At first, assume a good will "signaling" (CHC) strategy in the simultaneous hard close auction $(0 \leq$ $\left.b_{i j} \leq v_{i j}\right)$ :

- If $v_{i 1}>v_{i 2}$ submit $b_{i 1}^{1}=b_{i 1}$ and $b_{i 2}^{1}=\omega$;

- If $v_{i 1}<v_{i 2}$ submit $b_{i 1}^{1}=\omega$ and $b_{i 2}^{1}=b_{i 2}$;

- If, in stage 2 ,

$b_{x 1}^{1}=\omega$ and $b_{y 1}^{1} \geq 0$ and $b_{x 2}^{1} \geq 0$ and $b_{y 2}^{1}=\omega$

or

$b_{x 1}^{1} \geq 0$ and $b_{y 1}^{1}=\omega$ and $b_{x 2}^{1}=\omega$ and $b_{y 2}^{1} \geq 0$

the bidders divide the markets among each other and submit $b_{i 1}^{2}=v_{i 1}$ if $v_{i 1}>v_{i 2}$ and $b_{i 2}^{2}=v_{i 2}$ if $v_{i 1}<v_{i 2}$;

- If, in stage $2, b_{x j}^{1}=b_{y j}^{1}$ or if someone defects from the bidding instructions given above, then all types revert to the SSC strategy.

This CHC strategy equals the CSC strategy in that the bidders signal their favorite market and bidders can divide the markets according to their highest valuations. However, it is implemented such that bidders in the second stage submit their valuation in their preferred market. This makes defection of the other bidder more expensive and is therefore superior to submitting a final bid $b_{0}$ as in the collusion strategy of BL.

${ }^{\mathrm{i}}$ We do not consider side payments or repeated interaction. 
Contrary to the CHC strategy assume a sniping (SHC) strategy in the simultaneous hard close auction $\left(0 \leq b_{i j} \leq v_{i j}\right)$ :

- If $v_{i 1}>v_{i 2}$ submit $b_{i 1}^{1}=b_{i 1}$ and $b_{i 2}^{1}=\omega$;

- If $v_{i 1}<v_{i 2}$ submit $b_{i 1}^{1}=\omega$ and $b_{i 2}^{1}=b_{i 2}$;

- Play the SSC strategy in stage 2

In this strategy the bidders signal a willingness to collude in the first stage and indicate their full willingness to pay in each market in the second stage.

Considering these two strategies, this situation is a prisoner's dilemma with $C=$ Cooperation, i.e. playing the $\mathrm{CHC}$ strategy, and $D=$ Defection, i.e. playing the SHC strategy. Mutual cooperation and mutual defection leads to the same results as in Part 3.1. Playing $D$ while the other bidder plays $C$ potentially yields additional gains from the less preferred market, whereas playing $C$ while the other bidders plays $D$ potentially yields zero gains from their own preferred market. The payoffs for bidder $x$ depend on strategy combinations $\left(S_{x} ; S_{y}\right)$ where $S_{i}$ is either $C$ or $D$ and are (recall the assumption of the bidders' valuations):

$$
\begin{aligned}
E\left[\pi_{x}(\mathrm{CD})\right] & =\int_{0}^{v_{x 1}}\left(v_{x 1}-v_{y 1}\right) 2 f\left(v_{y 1}\right)\left(1-F\left(v_{y 1}\right)\right) d v_{y 1}, \\
E\left[\pi_{x}(\mathrm{DD})\right] & =E\left[\pi_{x}(S S C)\right], \\
E\left[\pi_{x}(\mathrm{CC})\right] & =v_{x 1}, \\
\text { and } E\left[\pi_{x}(\mathrm{DC})\right] & =v_{x 1}+\int_{0}^{v_{x 2}}\left(v_{x 2}-v_{y 2}\right) 2 f\left(v_{y 2}\right) F\left(v_{y 2}\right) d v_{y 2} .
\end{aligned}
$$

The payoffs for bidder $y$ depending on strategy are

$$
\begin{aligned}
E\left[\pi_{y}(\mathrm{CD})\right] & =\int_{0}^{v_{y 2}}\left(v_{y 2}-v_{x 2}\right) 2 f\left(v_{x 2}\right)\left(1-F\left(v_{x 2}\right)\right) d v_{x 2}, \\
E\left[\pi_{y}(\mathrm{DD})\right] & =E\left[\pi_{y}(S S C)\right], \\
E\left[\pi_{y}(\mathrm{CC})\right] & =v_{y 2}, \\
\text { and } \quad E\left[\pi_{y}(\mathrm{DC})\right] & =v_{y 2}+\int_{0}^{v_{y 1}}\left(v_{y 1}-v_{x 1}\right) 2 f\left(v_{x 1}\right) F\left(v_{x 1}\right) d v_{x 1} .
\end{aligned}
$$

Using a normal form matrix reveals a prisoner's dilemma situation with expected profits as Table 1 shows.

Defection is a dominant strategy since $\mathrm{IV}_{i}>\mathrm{III}_{i}$ and $\mathrm{II}_{i}>\mathrm{I}_{i}$. Hence, the $\mathrm{SHC}$ strategy is the best response to both given strategies. This profile forms a perfect Bayesian equilibrium (with a consistent belief system), i.e. if the probability that the competitor plays the $\mathrm{CHC}$ strategy $(p)$ is positive, the best response is to play $\mathrm{SHC}$ for $0 \leq p \leq 1$. In equilibrium the expected payoff in the simultaneous hard close auction equals the expected payoff in the perfect Bayesian equilibrium in SSC 
Table 1. Prisoner's Dilemma payoff matrix.

\begin{tabular}{lllclc}
\hline & \multicolumn{4}{c}{ Bidder $y$} \\
\cline { 3 - 6 } & & \multicolumn{2}{c}{$C H C$} & \multicolumn{2}{c}{$S H C$} \\
\hline \multirow{2}{*}{ Bidder $x$} & $C H C$ & $\mathrm{III}_{x}$ & $\mathrm{III}_{y}$ & $\mathrm{I}_{x}$ & $\mathrm{IV}_{y}$ \\
& $S H C$ & $\mathrm{IV}_{x}$ & $\mathrm{I}_{y}$ & $\mathrm{II}_{x}$ & $\mathrm{II}_{y}$ \\
\hline
\end{tabular}

with $\mathrm{IV}_{i}>\mathrm{III}_{i}>\mathrm{II}_{i}>\mathrm{I}_{i}$.

strategies in the simultaneous soft close auction. ${ }^{j}$ The seller receives the highest payoff and full efficiency is guaranteed.

Obviously, this game has further equilibria. All strategies that lead to bidding the valuation in both markets at least in the second stage forms an equilibrium. One equilibrum with asymmetric strategies is bidding for the object if the valuation is the highest in the market and not bidding otherwise.

\section{Conclusion}

In simultaneous English auctions (soft close auctions) with multiple objects, a collusive equilibrium where bidders reduce their payments exists (Brusco and Lopomo [2002]). This equilibrium holds due to the fact that defection from a collusive agreement can be punished later on because the English auction ends if no bidder wants to submit a bid. This collusive equilibrium in simultaneous hard close auctions, however, cannot be sustained even with only two bidders. In this auction format, the best response to a collusive strategy is to play a sniping strategy, i.e. signal to collude early in the auction but defect from collusion at the very last moment; giving the competitor no chance to react due to the deadline. Hence, in equilibrium the sniping strategy prevails. The situation equals a Prisoner's Dilemma situation because both bidders may increase their payoffs with mutual cooperation.

This result is due to the fact that the hard close auction has a fixed bidding deadline. As the number of bidders and the number of objects increase, coordination possibilities decrease and this advantage of the simultaneous hard close auction diminishes.

\section{Acknowledgments}

I thank Tim Hoppe, Tibor Neugebauer, Abdolkarim Sadrieh, and two anonymous referee for helpful comments.

\section{References}

Ariely, D., Ockenfels, A. and Roth, A. E. [2005] An experimental analysis of ending rules in internet auctions, The RAND Journal of Economics 36(4), 890-907. 
Bajari, P. and Hortaçsu, A. [2003] Winner's curse, reserve prices and endogenous entry: Empirical insights from eBay auctions, The RAND Journal of Economics 34(2), 329-355.

Brusco, S. and Lopomo, G. [2002] Collusion via signaling in simultaneous ascending bid auctions with heterogeneous objects, with and without complementarities, Review of Economic Studies 69(2), 407-436.

Engelberg, J. and Williams, J. [forthcoming] eBay's proxy bidding: A license to shill, Journal of Economic Behavior \& Organization 72(1) 509-526.

Engelbrecht-Wiggans, R. and Kahn, C. [2005] Low-revenue equilibria in simultaneous ascending-bid auctions, Management Science 51(3), 508-518.

Füllbrunn, S. and Neugebauer, T. [2009] Anonymity deters collusion in hard-close auctions: Experimental evidence, New Zealand Economics Papers 43(3), 131-148.

Füllbrunn, S. and Sadrieh, A. [2006] Sudden termination auctions - An experimental study, Journal of Economics and Management Study.

Myerson, R. [1981] Optimal auction design, Mathematics of Operations Research 6, 58-73.

Kwasnica, A. M. and Sherstyuk, K. [2007] Collusion and equilibrium selection in auctions, Economic Journal 117(516), 120-145.

Ockenfels, A. and Roth, A. E. [2006] Late and multiple bidding in second price internet auctions: Theory and evidence concerning different rules for ending an auction, Games and Economic Behavior 55(2), 297-320.

Ockenfels, A., Reiley, D. and Sadrieh, A. [2006] Online auctions, in Hendershott, T. (ed.), Economics and Information Systems, Amsterdam: Elsevier, 571-628.

Rasmusen, E. B. [2006] Strategic implications of uncertainty over one's own private value in auctions, Advances in Theoretical Economics 6(1), 1261-1261.

Rothkopf, M., Teisberg, T. and Kahn, E. [1990] Why are vickrey auctions rare? Journal of Political Economy 98(1), 94-109.

Vickrey, W. [1961] Counterspeculation, auctions, and competitive sealed tenders, The Journal of Finance 16(1), 8-37.

Wintr, L. [2008] Some evidence on late bidding in eBay auctions, Research series 200801-25, National Bank of Belgium. 